\title{
Critical phenomena in higher dimensional spaces: The hexagonal-to-orthorhombic phase transition in aperiodic $n$-nonadecane/urea
}

\author{
C. Mariette, ${ }^{1}$ L. Guérin, ${ }^{1}$ P. Rabiller, ${ }^{1}$ C. Ecolivet,,${ }^{1}$ P. García-Orduña, ${ }^{2}$ P. Bourges, ${ }^{3}$ A. Bosak, ${ }^{4}$ D. de Sanctis, ${ }^{4}$ \\ Mark D. Hollingsworth, ${ }^{5}$ T. Janssen, ${ }^{6}$ and B. Toudic ${ }^{1, *}$ \\ ${ }^{1}$ Institut de Physique de Rennes, UMR UR1-CNRS 6251, Université de Rennes 1, 35042 Rennes, France \\ ${ }^{2}$ Instituto de Síntesis Química y Catálisis Homogénea (ISQCH), CSIC - Universidad de Zaragoza, \\ Departamento de Química Inorgánica, Pedro Cerbuna 12, 50009 Zaragoza, Spain \\ ${ }^{3}$ Laboratoire Léon Brillouin, CEA-CNRS, CE Saclay, 91191 Gif-sur-Yvette, France \\ ${ }^{4}$ European Synchrotron Radiation Facility, BP 220, 38043 Grenoble Cedex, France \\ ${ }^{5}$ Department of Chemistry, 213 CBC Building, Kansas State University, Manhattan, Kansas 66506-0401, USA \\ ${ }^{6}$ Institute for Theoretical Physics, University of Nijmegen, Nijmegen, The Netherlands
}

(Received 29 November 2012; revised manuscript received 13 February 2013; published 4 March 2013)

\begin{abstract}
Upon cooling, the aperiodic inclusion compound $n$-nonadecane/urea presents a hexagonal-to-orthorhombic group-subgroup phase transition at $T_{\mathrm{cl}}$ that increases the structure's superspace dimensionality from four to five. This paper reports on pretransitional phenomena in such a high-dimensional space, generalizing the critical results previously reported at a lower dimensionality. Very high-resolution diffraction data reveal anomalously large correlation lengths along the aperiodic direction, with all correlation lengths diverging at $T_{\mathrm{cl}}$. This could be explained by low-frequency phason excitations that soften at $T_{\mathrm{cl}}$ at the critical wave vector, in accordance with an increase in the critical diffuse scattering intensity.
\end{abstract}

DOI: 10.1103/PhysRevB.87.104101

PACS number(s): 61.44.Fw, 64.60.-i, 61.05.cf

The physics of phase transitions in crystalline materials was studied extensively during the last decades of the 20th century. With respect to group-subgroup structural instabilities, the order parameters, as measures of symmetry breaking, as well as related critical phenomena, were derived and measured. ${ }^{1,2}$ Three-dimensional (3D) lattice periodicities of the crystals provided for a huge simplification, since all of the properties could be defined within the Brillouin zone. In this context, the concept of the soft-mode phonon was successfully developed to describe numerous structural instabilities that yield periodic or even quasiperiodic (incommensurately modulated) lowsymmetry phases. ${ }^{3}$ Because they do not possess a Brillouin zone, however, there are still no such simple descriptions of the critical phenomena that exist in materials that are aperiodic by construction.

Aperiodic crystals, such as incommensurate composites, recover translational symmetry in spaces of dimensions larger than three. ${ }^{4,5}$ Crystallographic superspace groups of rank $(3+d)$ describe these materials, in which group-subgroup phase transitions may occur either by maintaining the dimensionality of the superspace or by lowering or raising it. ${ }^{6}$ This last case was recently reported in a phase transition in $n$-nonadecane/urea, where the dimensionality increased from four to five. ${ }^{7,8}$ Although the translational symmetry in aperiodic crystals is recovered by using superspace crystallography, the study of collective excitations, including pretransitional phenomena, in such materials is presently in its infancy. ${ }^{6}$ We do know, however, that in $(3+d)$ dimensional superspaces, $d$ supplementary Goldstone-like branches, called phasons, exist as fluctuations along the $d$ internal dimensions of the superspace. ${ }^{2,4}$ Therefore, in phase transitions resulting from a soft mode, not only phonon modes but also phason modes might be involved in triggering the structural changes. Here, we report the critical phenomena leading to the $4 \mathrm{D}$-to-5D phase transition in the aperiodic composite $n$-nonadecane/urea. We interpret them in terms of a low-frequency excitation along the aperiodic direction that condenses at the transition temperature.

Aperiodic composites arise from the imbrication of two or more incommensurate substructures in which interactions of the components give rise to mutual incommensurate modulations. ${ }^{4,5,9,10}$ For uniaxial composites, such as host-guest systems, there is a single incommensurate direction $(\boldsymbol{c})$, and the reciprocal image is characterized by a four-dimensional superspace description,

$$
\mathbf{Q}_{\mathbf{h k l m}}=h \mathbf{a}_{\mathbf{o}}^{*}+k \mathbf{b}_{\mathbf{o}}^{*}+l \mathbf{c}_{\mathbf{h}}^{*}+m \mathbf{c}_{\mathrm{g}}^{*},
$$

in which $\mathbf{a}_{\mathbf{o}}^{*}, \mathbf{b}_{\mathbf{o}}^{*}, \mathbf{c}_{\mathbf{h}}^{*}$, and $\mathbf{c}_{\mathbf{g}}^{*}$ are the conventional reciprocal unit cell vectors, with $c_{h}$ and $c_{g}$ referring, respectively, to the host and guest repeat distances along the aperiodic direction. The ratio $c_{h} / c_{g}$ is defined as the misfit parameter $\gamma$. Here, four indices are needed to describe four different types of Bragg peaks $(h \mathrm{k} l \mathrm{~m}$ ). A convenient but simplistic labeling is that $(h \quad k \quad 00),(h k l 0),(h k 0 m)$, and $(h k l m)$, with $l$ and $m$ different from zero, are the common, host, guest, and satellite Bragg peaks, respectively, with the recognition that both substructures contribute to each kind of Bragg peak due to the aforementioned interactions. Prototypical examples of such aperiodic composites are given by the $n$-alkane/urea inclusion compounds. ${ }^{11-14}$ In these supramolecular systems, urea molecules form a helical, hydrogen-bonded network of parallel, hexagonal channels with internal diameters of $0.55-0.58 \mathrm{~nm} .{ }^{15}$ Such compounds exhibit the features of incommensurate composites since the repeat distances of host and guest along the channel axis $\left(c_{h}\right.$ and $c_{g}$ ) do not satisfy the relation $q c_{h}=r c_{g}$ for reasonably small integers $q$ and $r .{ }^{16}$ A large body of work has been dedicated to the phase transitions in this prototype family, but almost all treatments of these systems have ignored the role of aperiodicity. ${ }^{17-27}$ 


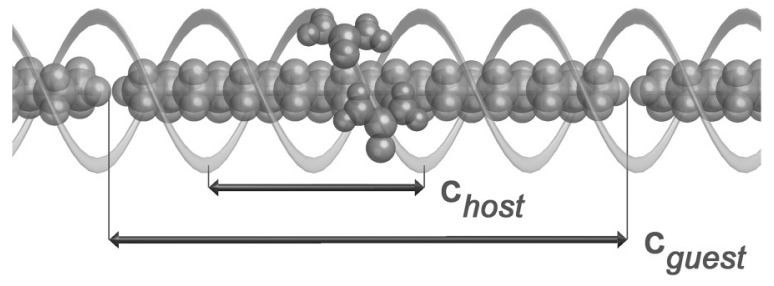

FIG. 1. A schematic representation of $n$-nonadecane/urea viewed perpendicular to the channel axis, revealing the urea host and the alkane guest periodicities.

This was the case in a previous study of the diffuse scattering in a different urea inclusion compound, $n$-hexadecane/urea. ${ }^{26}$

According to the superspace description of an $n$-alkane/urea aperiodic intergrowth structure, the parent phase (phase I) is described by the four-dimensional superspace group $P 6_{1} 22(00 \gamma) .{ }^{11,12}$ Within the construction of four-dimensional superspace, group-subgroup phase transitions have been discussed in detail by van Smaalen and Harris from a theoretical point of view. ${ }^{12}$ Recent studies have revealed, however, that the ferroelastic phase transition in $n$-nonadecane/urea (Fig. 1, with a misfit parameter $\gamma=0.418$ ) was beyond the scope of this discussion, since a transition from 4D to 5D superspace must be considered when describing the symmetry breaking in this system. ${ }^{7,8}$ According to this study, the ordered phase II, which has superspace group $C 222_{1}(00 \gamma)(10 \delta)$, displays a mean ferro-ordering of the host substructure as projected onto the (a, b) plane; antiferro-ordering (i.e., an alternation from channel to channel) does exist, but it concerns only the host-guest intermodulation (Fig. 2). The order parameter associated with phase II is a complex number, $\eta=A e^{i \phi}$, with (a)

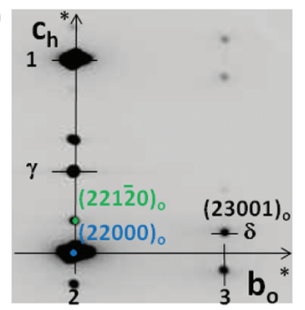

(b)

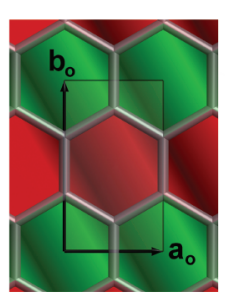

(c)

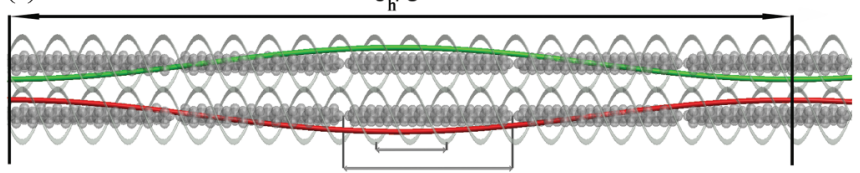

FIG. 2. (Color online) $n$-Nonadecane/urea low-symmetry phase II in reciprocal space and real space: (a) high-resolution reconstructed reciprocal plane $\left(2, \mathbf{b}_{\mathbf{0}}^{*}, \mathbf{c}_{\mathbf{h}}^{*}\right)$ at $T_{\mathrm{cl}}-4 \mathrm{~K}$, showing a basic structure line (with $h+k$ even) and a superstructure line (with $h+k$ odd) extending along the incommensurate direction $\mathbf{c}^{*}$ and indexed in host urea units, as measured by synchrotron diffraction of fully hydrogenated $n$-nonadecane/urea $\left(T_{\mathrm{cl}}=158.5 \mathrm{~K}\right)$. (b) Schematic representation in the $(\mathbf{a}, \mathbf{b})$ plane: the red and green antiphase modulations shown in (c) are represented by red and green regions. (c) Schematic representation of the ordering along the aperiodic direction $\mathbf{c}$, in which the intermodulation with period $c_{h} / \delta$ is phase shifted by $\pi$ from channel to channel. Here, $\gamma=c_{h} / c_{g}=0.418$, and $\delta=0.090$

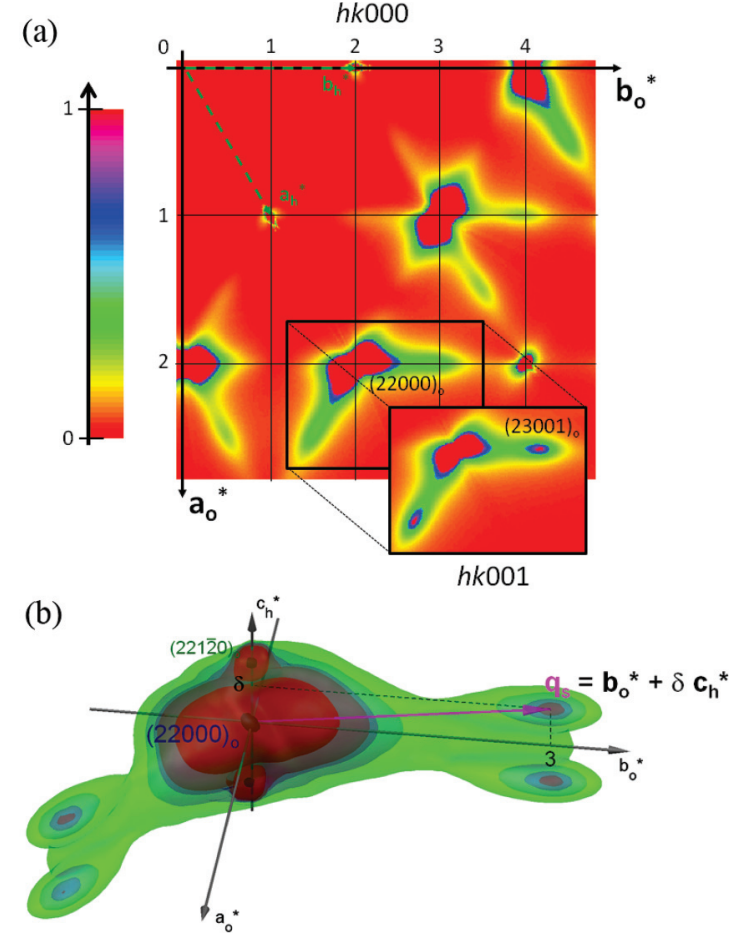

FIG. 3. (Color online) The critical diffuse scattering in the hightemperature phase as measured by very high-resolution synchrotron diffraction at $T_{\mathrm{cl}}+4 \mathrm{~K}\left(T_{\mathrm{cl}}=158.5 \mathrm{~K}\right)$ in fully hydrogenated

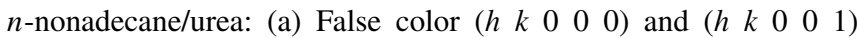
diffraction planes using the 5D low-symmetry notation; (b) False color reconstructed three-dimensional image of this pretransitional diffuse scattering, as seen from a general direction; the color palette goes from green to brown as intensities increase.

$A$ and $\varphi$ defining, respectively, the amplitude and the phase of this symmetry-breaking modulation. ${ }^{3,4}$ In the high-symmetry phase, all channels are equivalently modulated, whereas in the ordered phase II, a phase shift of $\pi$ relates the intermodulation in adjacent channels along $\mathbf{b}_{\mathbf{0}}$ [Figs. 2(b) and 2(c)]. ${ }^{8}$ In the following discussion, the low-symmetry notation will be used to assign the different Bragg peaks, even in the high-symmetry phase. Five indices $(h k l m n)_{\mathrm{o}}$ defined in the orthorhombic basis $\left(\mathbf{a}_{\mathbf{o}}^{*}, \mathbf{b}_{\mathbf{o}}^{*}, \mathbf{c}_{\mathbf{h}}^{*}, \mathbf{c}_{\mathbf{g}}^{*}\right.$, and $\left.\delta \mathbf{c}_{\mathbf{h}}^{*}\right)$ are thus associated with each Bragg peak. The critical wave vector is $\mathbf{q}_{\mathbf{s}}=\mathbf{b}_{\mathbf{o}}^{*}+\delta \mathbf{c}_{\mathbf{h}}^{*}$, which is along $\left(\begin{array}{lllll}0 & 1 & 0 & 0 & 1\end{array}\right)$. The value of $\delta$ was found to be $0.090 \pm 0.005$ (Refs. 7 and 8), as shown in Fig. 2(a).

In order to analyze the critical diffuse scattering, highresolution neutron and $\mathrm{x}$-ray diffraction techniques were used. Neutron studies were performed on the triple-axis spectrometer 4F located on the cold source at the Laboratoire Léon Brillouin. Very high-resolution synchrotron diffraction measurements were done at beam line ID 29 at the European Synchrotron Radiation Facility (ESRF) ${ }^{28}$ with a PILATUS $6 \mathrm{M}$ detector. ${ }^{29}$ There, the sample-to-detector distance was $150 \mathrm{~mm}$, and the incident wavelength was $0.6895 \AA$. Reciprocal planes were reconstructed using XCAVATE software. ${ }^{30}$ Data were averaged over Laue symmetry to improve the signal-to-noise ratio and to remove the artifacts related to the gaps in the detector. Single crystals of $n$-nonadecane/urea were prepared by slow evaporation of a solution of urea and $n$-nonadecane in a mixture of ethanol and isopropyl alcohol. 

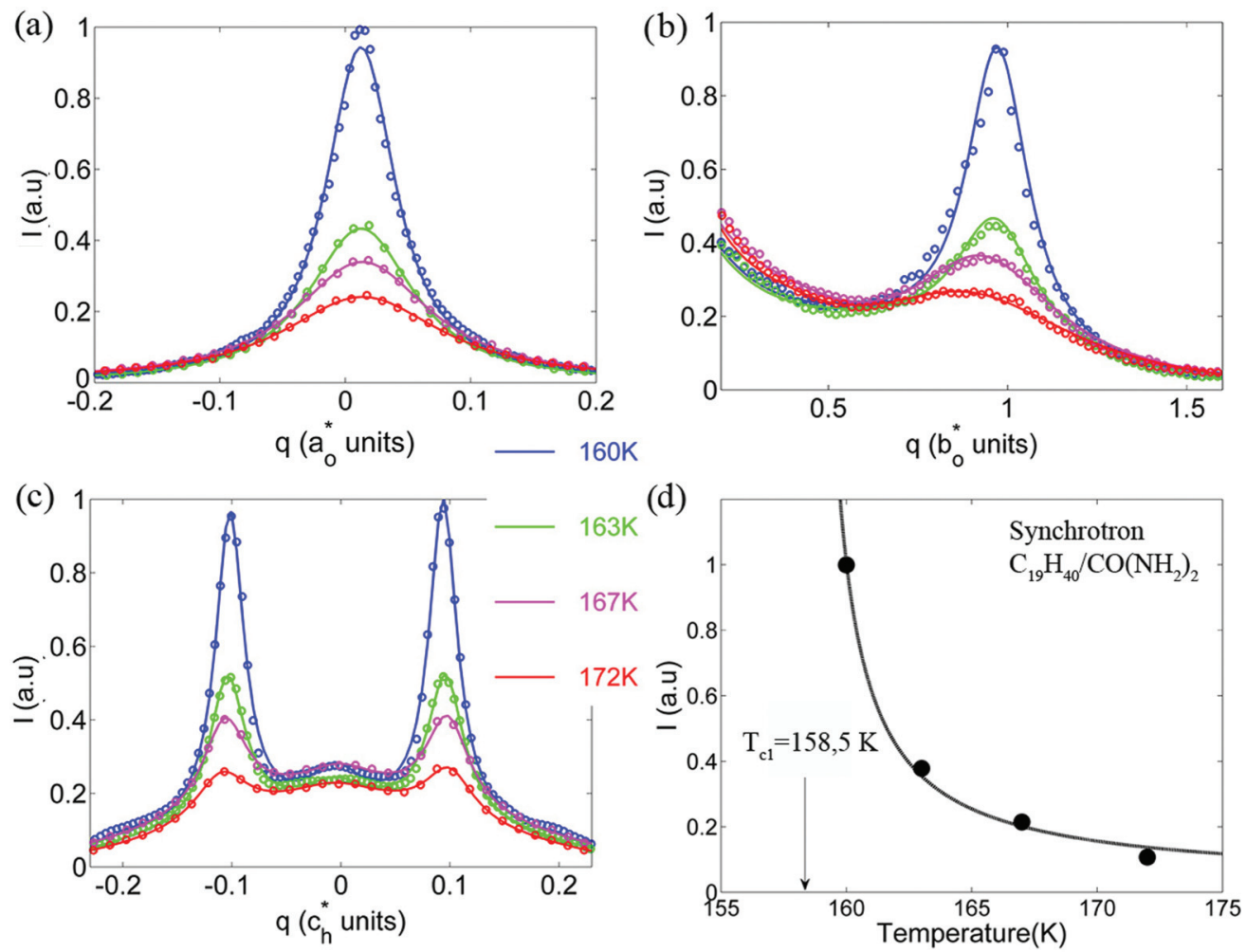

FIG. 4. (Color online) Temperature dependence of the diffuse scattering associated with the critical wave vector $\left(\begin{array}{lllllllll}0 & 0 & 0 & 1\end{array}\right)$ along the directions (a) $\mathbf{a}_{\mathbf{0}}^{*}$, (b) $\mathbf{b}_{\mathbf{0}}^{*}$, and (c) $\mathbf{c}^{*}$, as measured in fully hydrogenated $n$-nonadecane/urea at $160 \mathrm{~K}$ (blue), $163 \mathrm{~K}$ (green), $167 \mathrm{~K}$ (pink), and $172 \mathrm{~K}$ (red). The asymmetric background along $\mathbf{b}^{*}$ and the broad quasielastic component along $\mathbf{c}^{*}$ arise from diffuse scattering emerging from

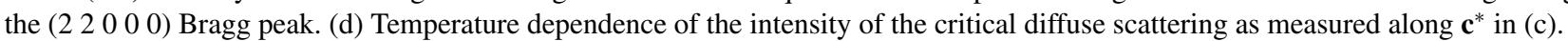

In order to obtain a full description of the static pretransitional phenomena in the three directions of physical space, complete $\mathrm{x}$-ray data acquisitions were performed with nonaligned crystals of fully hydrogenated $n$-nonadecane/urea.

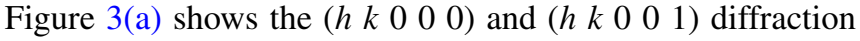
planes as determined from synchrotron diffraction measurements, using a pseudocolor palette. These figures show a rather complex pattern. The diffuse scattering reaches a maximum in the $(h k 000)$ diffraction plane, appearing as a peanut shape around the strong Bragg peak, due mostly to low-frequency transverse acoustic (TA) phonons, whereas the pretransitional diffuse scattering (i.e., that not associated with a Bragg peak from the high-symmetry phase) is most intense in the ( $h k 001)$ plane. Figure 3(b) shows the isointensity surfaces of the diffuse scattering in three-dimensional reciprocal space. The analysis of this disc-shaped pretransitional x-ray diffuse scattering around the critical wave vector $\left(\begin{array}{lllll}0 & 1 & 0 & 0 & 1\end{array}\right)$ allows the determination of the ellipsoid associated with the correlation lengths $\left(\xi_{\mathrm{a}}, \xi_{\mathrm{b}}, \xi_{\mathrm{c}}\right)$, the principal axes of this ellipsoid being found along the directions $\mathbf{a}^{*}, \mathbf{b}^{*}$, and $\mathbf{c}^{*}$ of the orthorhombic reciprocal cell:

$$
S(q, T) \propto \frac{k_{B} T}{1+\xi_{a}^{2} q_{a}^{2}+\xi_{b}^{2} q_{b}^{2}+\xi_{c}^{2} q_{c}^{2}} .
$$

Slices along these directions give the profiles shown in Fig. 4. In each case, the correlation length was extracted by fitting the data with a Lorentzian convoluted with a Gaussian function that describes the same satellite Bragg peak measured in the ordered phase. It should be noted that the intensities of the transverse acoustic phonons that emerge from the strong (2 2000 ) Bragg peak have not vanished in the superstructure reciprocal region $(h+k=$ odd). This required a supplementary contribution to the background in the fitting procedures.

The extracted correlation lengths for the fully hydrogenated compound are shown in Fig. 5. These correlation lengths appear isotropic in the (a, b) plane in the limit of precision of the refinement, which is complicated by the contribution of

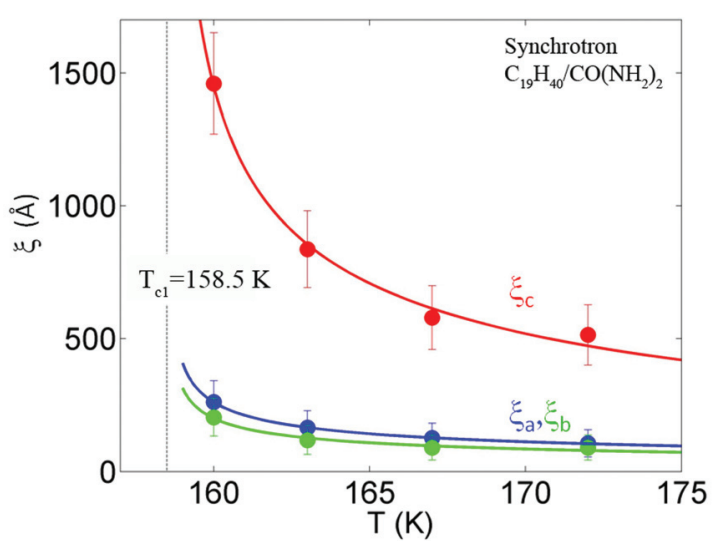

FIG. 5. (Color online) Temperature dependence of the correlation lengths $\xi_{\mathbf{a}}, \xi_{\mathbf{b}}$, and $\xi_{\mathbf{c}}$, respectively, along the directions $\mathbf{a}_{\mathbf{o}}$ (blue), $\mathbf{b}_{\mathbf{o}}$ (green), and $\mathbf{c}$ (red) in fully hydrogenated $n$-nonadecane/urea. The data are fit with a $\left(T-T_{\mathrm{cl}}\right)^{-v}$ law with $v=0.4$ and $T_{\mathrm{cl}}=158.5 \mathrm{~K}$. 

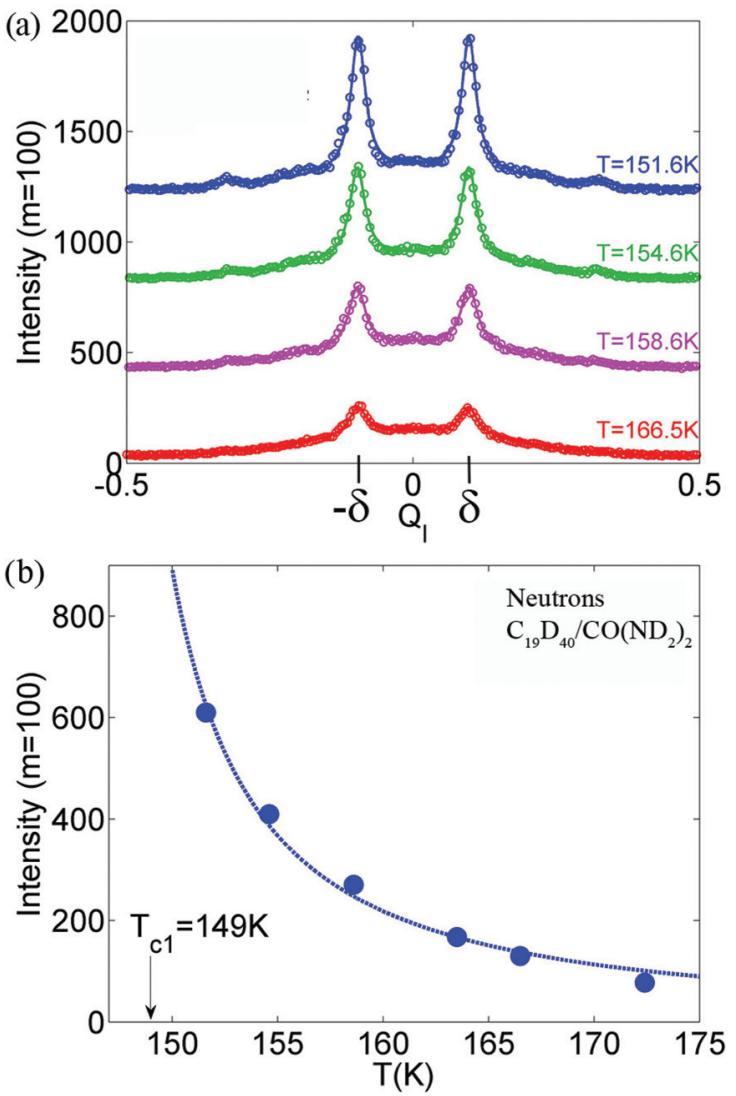

FIG. 6. (Color online) Temperature dependence of the diffuse scattering associated with the critical wave vector $\left(\begin{array}{lllll}0 & 1 & 0 & 0 & 1\end{array}\right)$ along $\mathbf{c}^{*}$ and referred to the Bragg (2 20000 ), as measured in fully deuterated $n$-nonadecane/urea at $151.6 \mathrm{~K}$ (blue), $153.6 \mathrm{~K}$ (green), 158.6 K (pink), and $166.5 \mathrm{~K}$ (red). The broad quasielastic component arises from diffuse scattering emerging from the (2 20000$)$ Bragg peak. (b) Temperature dependence of the intensity of the critical diffuse scattering as measured along $\mathbf{c}^{*}$.

other low-frequency phonons (mainly TA branches). Along the aperiodic direction $\mathbf{c}$, the extracted lengths are much larger. On approaching $T_{\mathrm{c} 1}$, they are very close to the experimental resolution limit even with this very high-resolution synchrotron diffractometer. The increase in the different correlation lengths as the temperature is lowered can be described by a critical power law $\left(T-T_{\mathrm{cl}}\right)^{-v}$, with $v=0.4 \pm 0.1$ and $T_{\mathrm{cl}}=158.5 \mathrm{~K}$. The pretransitional effects appear to be associated with quasione-dimensional fluctuations along the aperiodic direction $\mathbf{c}$ accompanied by a lateral ordering in the $(\mathbf{a}, \mathbf{b})$ plane upon approaching $T_{\mathrm{c} 1}$.

An equivalent study was performed by coherent neutron scattering on a fully deuterated crystal of $n$-nonadecane/urea, which revealed the original sequence of phases in this crystal. ${ }^{7,8}$ The best spatial resolution was used performing cold neutron diffraction measurements. The spectra measured

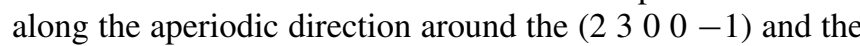
(2 3000 1) positions are reported in Fig. 6(a) at several temperatures in the high-symmetry phase. The temperature evolution of the integrated intensity measured at these critical wave vectors $\left(\mathbf{q}_{\mathbf{s}}=\mathbf{b}_{\mathbf{0}}{ }^{*}+\delta \mathbf{c}_{\mathbf{h}}\right)$ is shown in Fig. 6(b). According to these data, in agreement with the previous diffraction analysis, the transition temperature $T_{\mathrm{c} 1}$ in the fully deuterated compound is found to be $149 \pm 1 \mathrm{~K}$. This transition temperature depends on the isotopic substitution. Hydrogenation has the same effect as the pressure, ${ }^{8}$ that is, it increases the intermolecular potential and favors the low-symmetry phase. The isotope effect on the transition temperature is about $10 \mathrm{~K}$ for the 4D-hexagonalto-5D-orthorhombic phase transition. Nevertheless, the same value of the parameter $\delta=0.090$ is found in both materials. In the neutron measurements reported in Fig. 6(a), diffuse scattering along the aperiodic direction appears as Bragg peaks at all temperatures. These results are consistent with the ones reported by synchrotron diffraction in the fully hydrogenated $n$-nonadecane/urea since the actual correlation lengths are always larger than $500 \AA$, which is the typical resolution limit with the best neutron diffraction experiments.

The critical phenomena in both fully hydrogenated and fully deuterated compounds could be explained as a result of low-frequency excitations in the high-symmetry phase that soften at $T_{\mathrm{c} 1}$. This result is corroborated by the increase in the diffuse scattering intensity measured around $\mathbf{q}_{\mathrm{s}}$ [Figs. 4(d) and 6(b)]. Phase II would therefore appear to be the result of the condensation of the collective mode, in an analogous way reported in three-dimensional crystals when approaching incommensurately modulated phases. ${ }^{3}$ In the latter case, the softening concerns phonons, which are the only excitations present in the periodic high-symmetry phase. In a fourdimensional aperiodic composite, a phason branch is additionally present in the high-symmetry phase. The specific feature of the correlation lengths, almost infinite along the aperiodic direction, raises the possibility of a phase fluctuation leading to the antiferro-ordering along the supplementary internal space of the superspace. Simple models can be used to implicate either a phonon branch (acoustic or optical) or such a phason branch. ${ }^{31}$ However, future inelastic scattering experiments are needed to distinguish these branches; here the phason branch should be overdamped as the critical fluctuations lead to the five-dimensional structure. Below $T_{\mathrm{c} 1}$, a new hydrodynamic phason mode will appear, namely, the pseudo-Goldstone mode associated with the fifth dimension.

The experimental work reported here generalizes in higherdimensional space the treatment of pretransitional phenomena, which was previously elaborated for phase transitions that take periodic structures to either commensurate or incommensurate ones.

\footnotetext{
*Author to whom correspondence should be addressed: bertrand.toudic@univ-rennes1.fr

${ }^{1}$ E. M. Lifshitz and L. P. Pitaevskii, Physical Kinetics. Course of Theoretical Physics, Vol. 10 (Pergamon, Oxford, 1981).
} 
${ }^{4}$ T. Janssen, G. Chapuis, and M. de Boissieu, Aperiodic Crystals: From Modulated Phases to Quasicrystals (Oxford University Press, Oxford, 2007).

${ }^{5}$ T. Janssen, A. Janner, A. Looijenga-Vos, and P. M. de Wolff, in International Tables for Crystallography, edited by A. J. C. Wilson, Vol. C (Kluwer Academic, Dordrecht, 2006), p. 907.

${ }^{6}$ T. Janssen, Acta Crystallogr. A 68, 667 (2012).

${ }^{7}$ B. Toudic, P. Garcia, C. Odin, P. Rabiller, C. Ecolivet, E. Collet, P. Bourges, G. J. McIntyre, M. D. Hollingsworth, and T. Breczewski, Science 319, 69 (2008).

${ }^{8}$ B. Toudic, P. Rabiller, L. Bourgeois, M. Huard, C. Ecolivet, G. J. McIntyre, P. Bourges, T. Breczewski, and T. Janssen, Europhys. Lett. 93, 16003 (2011).

${ }^{9}$ P. Coppens, Acta Crystallogr. B 51, 402 (1995).

${ }^{10} \mathrm{~S}$. van Smaalen, Incommensurate Crystallography (Oxford University Press, Oxford, 2007).

${ }^{11}$ K. D. M. Harris and J. M. Thomas, J. Chem. Soc., Faraday Trans. 86, 2985 (1990).

${ }^{12}$ S. van Smaalen and K. D. M. Harris, Proc. R. Soc. London, Ser. A 452, 677 (1996).

${ }^{13}$ R. Lefort, J. Etrillard, B. Toudic, F. Guillaume, T. Breczewski, and P. Bourges, Phys. Rev. Lett. 77, 4027 (1996).

${ }^{14}$ T. Weber, H. Boysen, F. Frey, and R. B. Neder, Acta Crystallogr. B 53, 544 (1997).

${ }^{15}$ M. D. Hollingsworth and K. D. M. Harris, in Comprehensive Supramolecular Chemistry, edited by D. D. MacNicol, F. Toda, and R. Bishop (Elsevier Science, Oxford, 1996), p. 177.

${ }^{16}$ H. U. Lenné, H. C. Mez, and W. Schlenk, Jr., Justus Liebigs Ann. Chem. 732, 70 (1970).
${ }^{17}$ Y. Chatani, H. Anraku, and Y. Taki, Mol. Cryst. Liq. Cryst. 48, 219 (1978).

${ }^{18}$ R. Forst, H. Boysen, F. Frey, H. Jagodzinski, and C. Zeyen, J. Phys. Chem. Solids 47, 1089 (1986).

${ }^{19}$ R. Forst, H. Jagodzinski, H. Boysen, and F. Frey, Acta Crystallogr. B 43, 187 (1987).

${ }^{20}$ R. Forst, H. Jagodzinski, H. Boysen, and F. Frey, Acta Crystallogr. B 46, 70 (1990).

${ }^{21}$ K. Fukao, J. Chem. Phys. 92, 6867 (1990).

${ }^{22}$ K. Fukao, T. Horiuchi, S. Taki, and K. Matsushige, Mol. Cryst. Liq. Cryst. B 180, 405 (1990).

${ }^{23}$ K. D. M. Harris, I. Gameson, and J. M. Thomas, J. Chem. Soc., Faraday Trans. 86, 3135 (1990).

${ }^{24}$ A. J. O. Rennie and K. D. M. Harris, J. Chem. Phys. 96, 7117 (1992).

${ }^{25}$ R. M. Lynden-Bell, Mol. Phys. 79, 313 (1993).

${ }^{26}$ T. R. Welberry and S. C. Mayo, J. Appl. Crystallogr. 29, 353 (1996).

${ }^{27}$ T. Weber, H. Boysen, and F. Frey, Acta Crystallogr. B 56, 132 (2000).

${ }^{28}$ D. de Sanctis, A. Beteva, H. Caserotto, F. Dobias, J. Gabadinho, T. Giraud, A. Gobbo, M. Guijarro, M. Lentini, B. Lavault, T. Mairs, S. McSweeney, S. Petitdemange, V. Rey-Bakaikoa, J. Surr, P. Theveneau, G. A. Leonard, and C. Mueller-Dieckmann, J. Synchrotron Radiat. 19, 455 (2012).

${ }^{29}$ C. Brönnimann, E. F. Eikenberry, B. Henrich, R. Horisberger, G. Hülsen, E. Pohl, B. Schmitt, C. Schulze-Briese, M. Suzuki, T. Tomizaki, H. Toyokawa, and A. Wagner, J. Synchrotron Radiat. 13, 120 (2006).

${ }^{30}$ M. Estermann and W. Steurer, Phase Trans. 67, 165 (1998).

${ }^{31}$ T. Janssen, Ferroelectrics 412, 4 (2011). 